Send your research letters to the editor British Dental Journal, 64 Wimpole Street, London W1G8YS E-mail bdj@bda.org Priority will be given to letters less than 500 words long. Authors must sign the letter, which may be edited for reasons of space

\section{Severe tissue damage}

Sir, the article Severe tissue damage and neurological deficit following extravasation of sodium hypochlorite solution during routine endodontic treatment (BDJ 2005; 198: 749) refers to a case where hypochlorite has extravasated and caused various problems. There is no indication in the article as to why the solution did extravasate although the discussion 'presumes' 'forced pressure irrigation led to entry of sodium hypochlorite into the soft tissues'. There is nothing in the article to back up that presumption and indeed the description of the practitioner using rubber dam would, if anything, indicate a reasonable and proper approach to endodontics.

I am therefore concerned at the use of the term 'misuse' in the précis at the head of the article, as hypochlorite irrigation per se is not misuse even if solution does extrude. It can happen accidentally and the references at the end of the article refer to 'inadvertent injection' 'hypochlorite induced' and 'caused by'. None of these terms indicate that, in the absence of evidence of improper use of hypochlorite, there is 'misuse'.

Although thankfully infrequent, there are regular problems of extravasation and I would not want to see a claim of negligence backed by this article implying that any case qualifies as 'misuse'.

It is too late to change the word or add further to the précis but I wonder if Witton and Brennan would respond in your letter page to clarify this matter in public.

\section{B. Westbury}

By email

Authors of the paper P. Brennan and $R$. Witton respond: We thank Mr Westbury for his comments about our report. Sodium hypochlorite solution is widely used in endodontic practice and fortunately complications are very rare. The main purpose of the paper was to document the previously unreported facial nerve involvement (resulting in upper lip weakness), to serve as a reminder to the dental practitioner of the possible severe complications that can occur when this solution extrudes into soft tissues, and to discuss the immediate management.

We would concur with Mr Westbury that this complication can occur accidentally, and indeed in the opening 'in brief' section at the top of the paper, our second statement was that 'accidents, while rare, can result in severe sequelae'. Although there was no conclusive evidence for the cause of the extrusion in the case presented, it would seem likely that the hypochlorite solution entered the periapical tissues under increased pressure. This assumption was made on the basis of normal apical anatomy being confirmed on radiographs and the severe nature of the presentation.

Each and every case of hypochlorite extrusion needs to be assessed and managed on an individual basis. With that in mind, we would certainly not wish this article to be used to back a claim for negligence for all cases of hypochlorite extrusion, especially as we wrote in our paper several times that this complication can be accidental in nature.

Interestingly, since our report was published, we have treated another patient with hypochlorite extrusion, which caused rapid severe life threatening airway compromise, requiring emergency surgical decompression. ${ }^{1}$ Fortunately severe complications with hypochlorite extrusion are very rare indeed, with only a handful of publications in the world literature. We would reiterate that prompt recognition and referral is necessary to minimise the risk of further complications.

1. Bowden J R, Ethunandan M, Brennan PA. Life threatening airway obstruction secondary to hypochlorite extrusion during root canal treatment. Oral Surg Oral Med Oral Pathol 2005 (in press).

doi: $10.1038 / s j . b d j .4812823$

\section{Mixed messages}

Sir, the paper An outcome audit of the treatment of acute dentoalveolar infection:

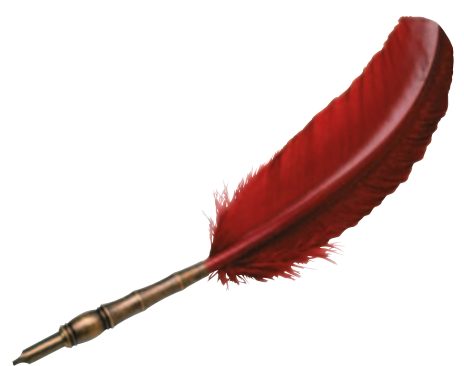

impact of penicillin resistance (BDJ 2005; 198: 759-763) is timely because of the increasing worldwide problems of antimicrobial resistance and the evidence of inappropriate and overuse of antibiotics in dental practice. ${ }^{1-3}$ What I found interesting was that only 112 patients were included in the audit period from January 1999 to January 2003.Is this an indication that there are very few incidences of acute dentoalveolar spreading infections in patients attending emergency clinics? I was also interested to see that only soft tissue incision and opening root canals were used and that no teeth were extracted to establish drainage in this group of patients.

The indications for prescribing antibiotics, with drainage, in the treatment of dentoalveolar infections are well defined in the literature, namely, raised body temperature, signs of spreading infection and local lymphadenopathy. In this audit a note of the presence of extraoral or intraoral swelling was recorded, but there was no measure of whether there were signs of a spreading infection and of the 112 cases only 17 had a raised temperature. I was also surprised that in a University Dental Hospital six different antibiotic regimens were used, some of which were outside the recommendations of the Dental Practitioners' Formulary. Is this further evidence of inappropriate antibiotic prescribing in dentistry?

The authors state in their results that all the antibiotics produced a satisfactory outcome, despite the presence of penicillin-resistant bacteria. Was it the drainage of the infection, was it the antibiotic or was it a combination of the two which produced the satisfactory outcome? The authors admit in their conclusions that they failed to address these questions and that in many of the cases studied antibiotics may not have been required.

This paper sends out mixed messages to dentists and does little to encourage more 
judicious use of antibiotics within

dentistry.

N. Palmer

Liverpool

doi: 10.1038/sj.bdj.4812824

1. Huovinen $\mathrm{P}, \mathrm{Cars}$ 0. Control of antimicrobial resistance: time for action. The essentials of control are already well known. BrMed J 1998; 317: 613-614.

2. Palmer N O A, Pealing R, Ireland R S, Martin M V. A study of therapeutic antibiotic prescribing in National Health Service general dental practice in England. Br Dent J 2000; 10: 554-558.

3. Palmer N O A, Martin M V, Pealing R, Ireland R S. An analysis of antibiotic prescriptions from general dental practitioners in England. J Antimicrob Chemother 2000; 446: 1033-1035.

\section{Patch test value}

Sir, we would like to congratulate Y. Issa et $a l$. for their paper in the recent issue of the BDJ: Oral lichenoid lesions related to dental materials (BDJ 2005; 198: 361). It provides further evidence of the role played by dental materials, and particularly amalgam, in some oral lichenoid lesions and confirms the value of replacing restorations that are in direct contact with the oral mucosa in such cases.

It is clear that amalgam replacement can be beneficial in patients with amalgam associated lichenoid lesions (OLL) but is unhelpful in cases of idiopathic oral lichen planus (OLP). The dilemma for most clinicians, however, is in clearly distinguishing these two conditions. Where there is only one amalgam restoration in contact with the oral mucosa and one adjacent lichenoid lesion then, in practice, the close topographical relationship between the lesion and restoration may make the decision to replace the one restoration straightforward. However, the real difficulty arises in patients with lots of amalgam restorations and a pattern of lesions that would be consistent with either diagnosis, a not uncommon situation. In such situations, anything that will improve the reliability of the diagnosis is important given the tissue destruction, high financial cost, time and inconvenience to the patient of replacing restorations, particularly if it is of no benefit.

The authors make reference to a study ${ }^{1}$ in which we concluded that 'A strong clinical association between lesions and amalgam restorations, plus a positive patch test, was a good predictor of lesion improvement on amalgam replacement' ie help distinguish cases of OLP from OLL. Although they agree with our conclusion about the importance of the topographical relationship, they criticise our conclusion about the value of patch testing. However, their criticism appears to be based on a misinterpretation of our results and requires a reply.

Our conclusion was based on the finding that only $3.9 \%$ of patients, whose lesions had little or no association with their restorations (ie more likely to be OLP) were patch test positive, compared to $70 \%$ of patients whose lesions had a strong or very strong association between their lesions and their restorations (ie more likely to be OLL). This difference was statistically significant $(p<0.0001)$. The paper by Issa et al. does not appear to have looked at the value of patch testing in distinguishing OLP from OLL.

What their study did indicate was that there can be a significant false negative rate when patients with apparent OLL are patch tested. In future, this is something that might presumably be improved with better knowledge of the allergens involved and better patch testing protocols.

We continue to believe that our data support the view that patch testing, in conjunction with the topographical relationship between the lesions and any amalgam restorations, combine in helping to distinguish these two conditions. Indeed, our data showed that the combination of a positive patch test and a strong/very strong clinical association between the lesions and the restorations was a better predictor of lesion improvement following amalgam replacement (with an NNT $=1.1$ [the number of patients with a positive test result that would need to be treated to result in one successful treatment outcome]) than either a positive patch test $(\mathrm{NNT}=2)$ or a strong/very strong clinical association alone (NNT $=$ I.46). Since the study by Issa et al. did not look at the value of patch testing in distinguishing OLL from OLP, we do not feel that their conclusion that patch testing is of limited value, or their criticism of our findings, is justified. Interested readers may like to read both papers and draw their own conclusions. M. H. Thornhill

\section{San Antonio, USA}

R. K. Simmons

Las Vegas, USA

M. N. Pemberton

E. D. Theaker

\section{Manchester}

1. Thornhill M H, Pemberton M N, Simmons R K, Theaker E D. Amalgam-contact hypersensitivity lesions and oral lichen planus. Oral Surg Oral Med Oral Pathol Oral Radiol Endod 2003; 95: 291-299.

The authors of the paper referred to did not provide a response to this letter.

doi: $10.1038 /$ sj.bdj.4812825 